\title{
A green route to grow hierarchical SAPO-34 crystal with excellent catalytic performance in methanol to olefin reaction
}

Yu-Rong He ${ }^{a}$, Ying-Liang Zhu ${ }^{a}$, Ying Duan ${ }^{a, c}$, Meng Zhang ${ }^{a},{ }^{*}$, Jiuxing Jiang ${ }^{b}$

a, School of Chemical Engineering and Technology, Sun Yat-sen University, Zhuhai 519082, Guangdong, PR China

b, School of Chemistry, Sun Yat-sen University, Guangzhou 510275, Guangdong, PR China

c, State Key Laboratory of Structural Chemistry,

Fujian Institute of Research on the Structure of Matter, Chinese Academy of Sciences, Fuzhou, Fujian 350002, PR China 


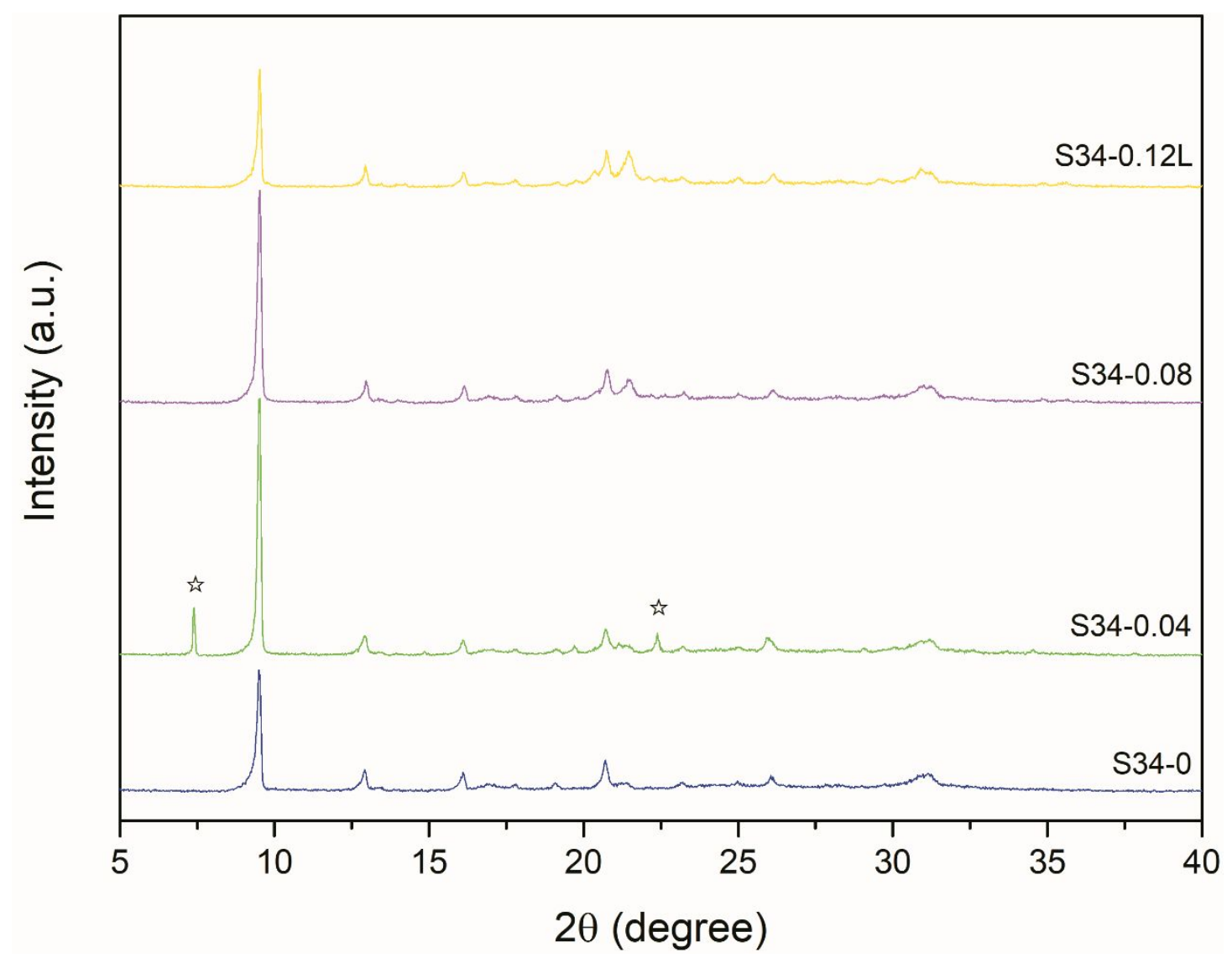

Fig. S1. XRD patterns of aluminosilicophosphate samples of S34-0, S34-0.04, S340.08 and S34-0.12L. is labels the peak of SAPO-5. 


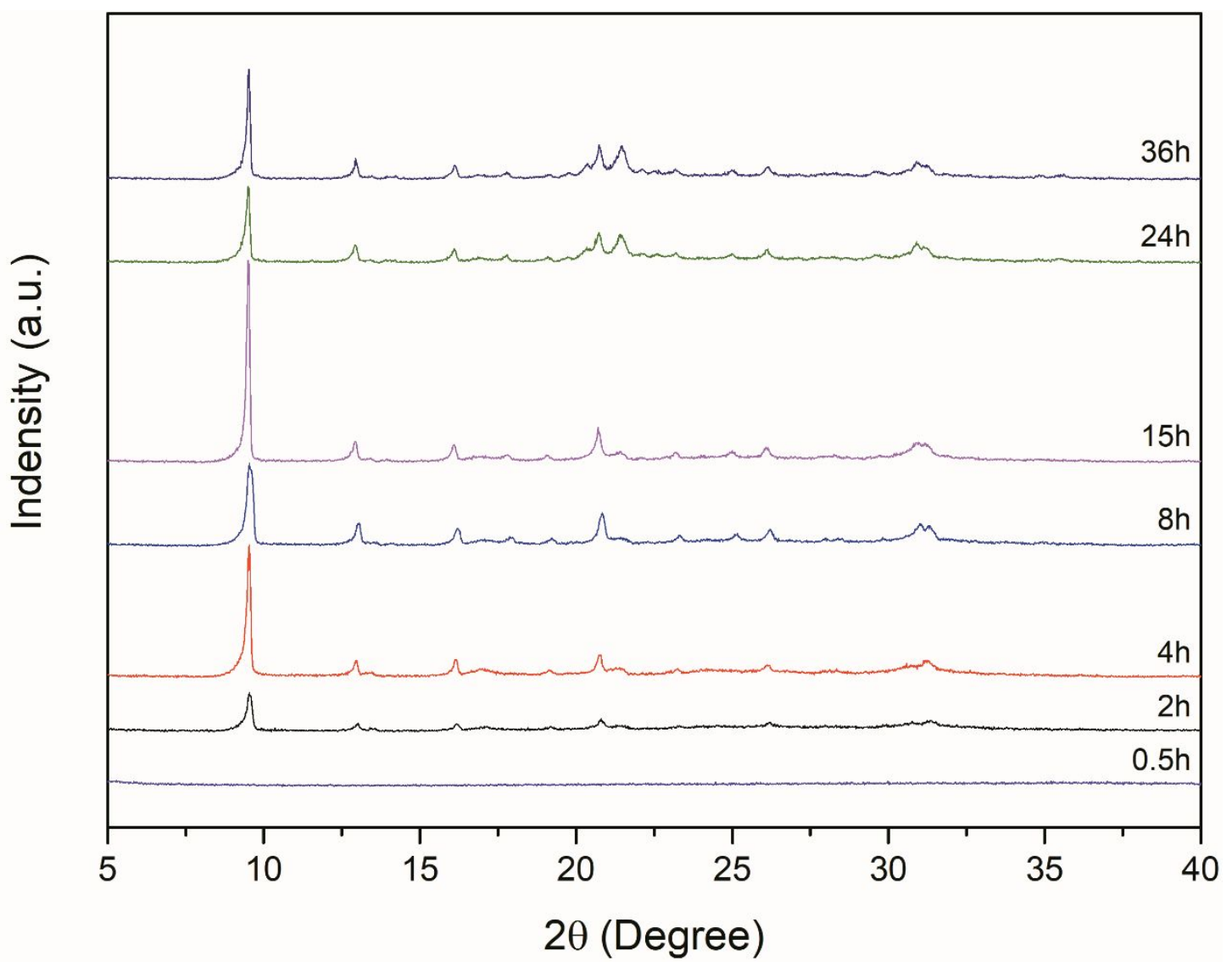

Fig. S2. XRD patterns of sample S34-0.12L with different crystallization time. 


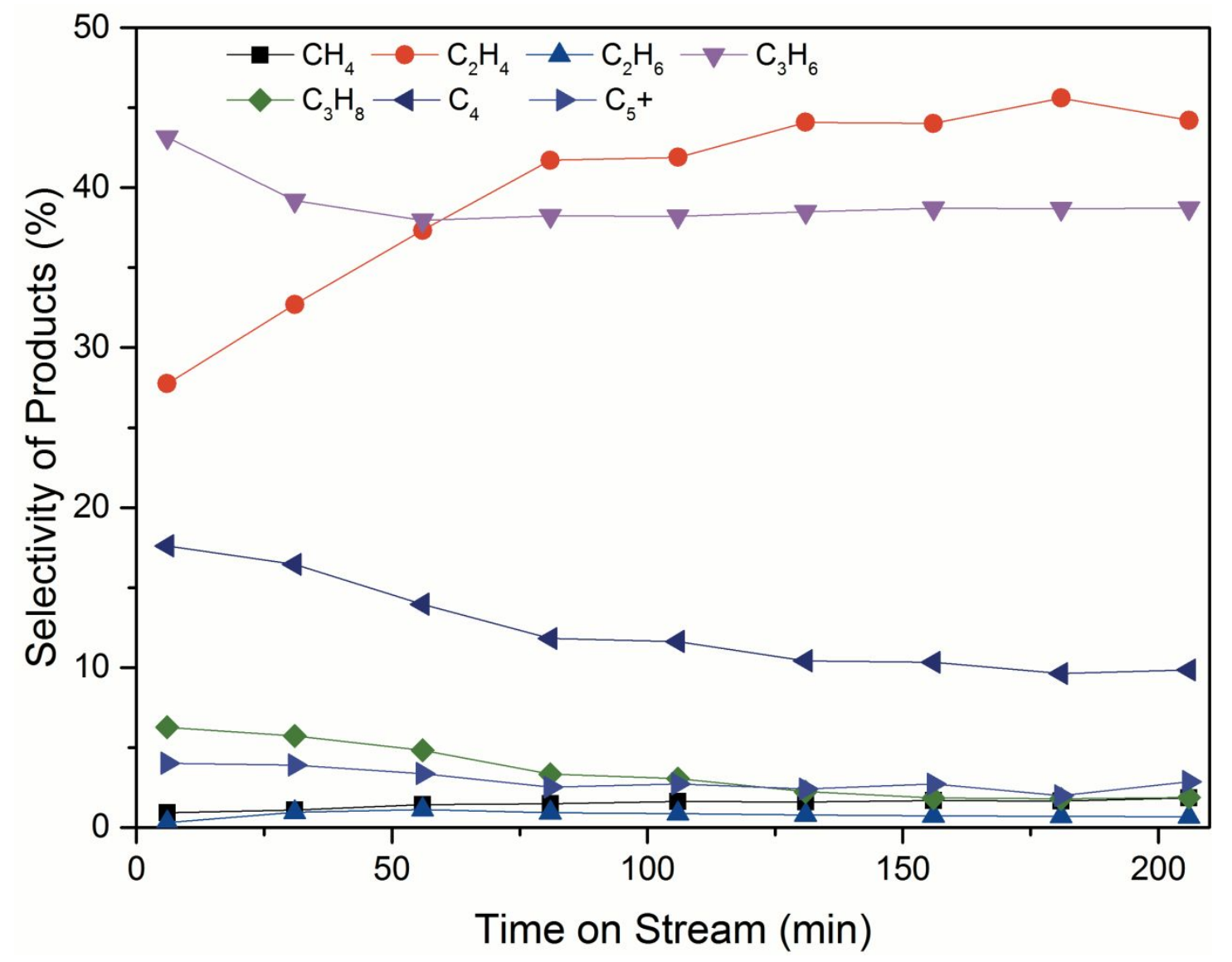

Fig. S3. Selectivity of hydrocarbons over S34-0 sample. Experimental conditions: $450{ }^{\circ} \mathrm{C}$, WHSV $=2 \mathrm{~h}^{-1}, 50 \%$ methanol solution. 


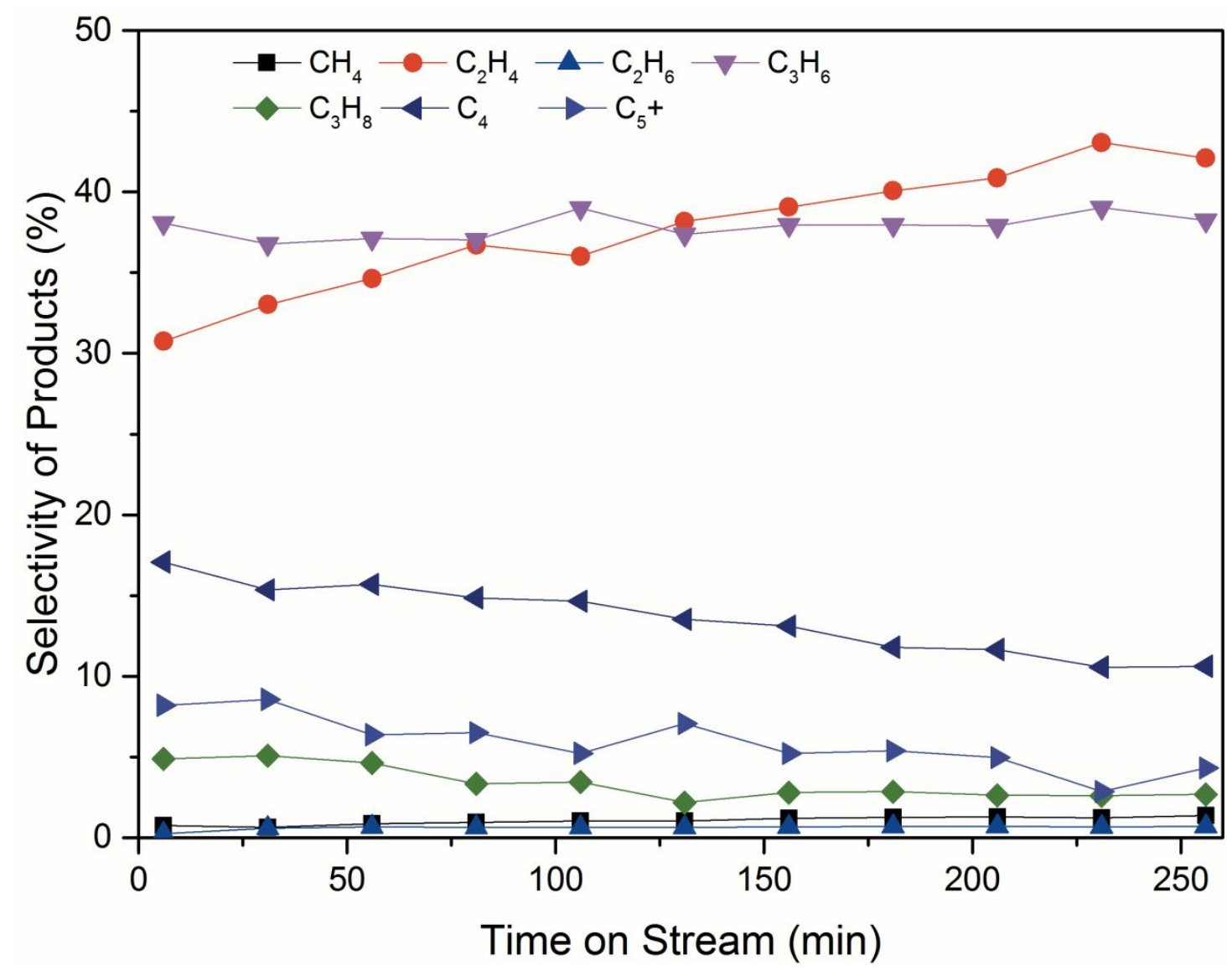

Fig. S4. Selectivity of hydrocarbons over S34-0.04 sample. Experimental conditions: $450{ }^{\circ} \mathrm{C}$, WHSV $=2 \mathrm{~h}^{-1}, 50 \%$ methanol solution. 


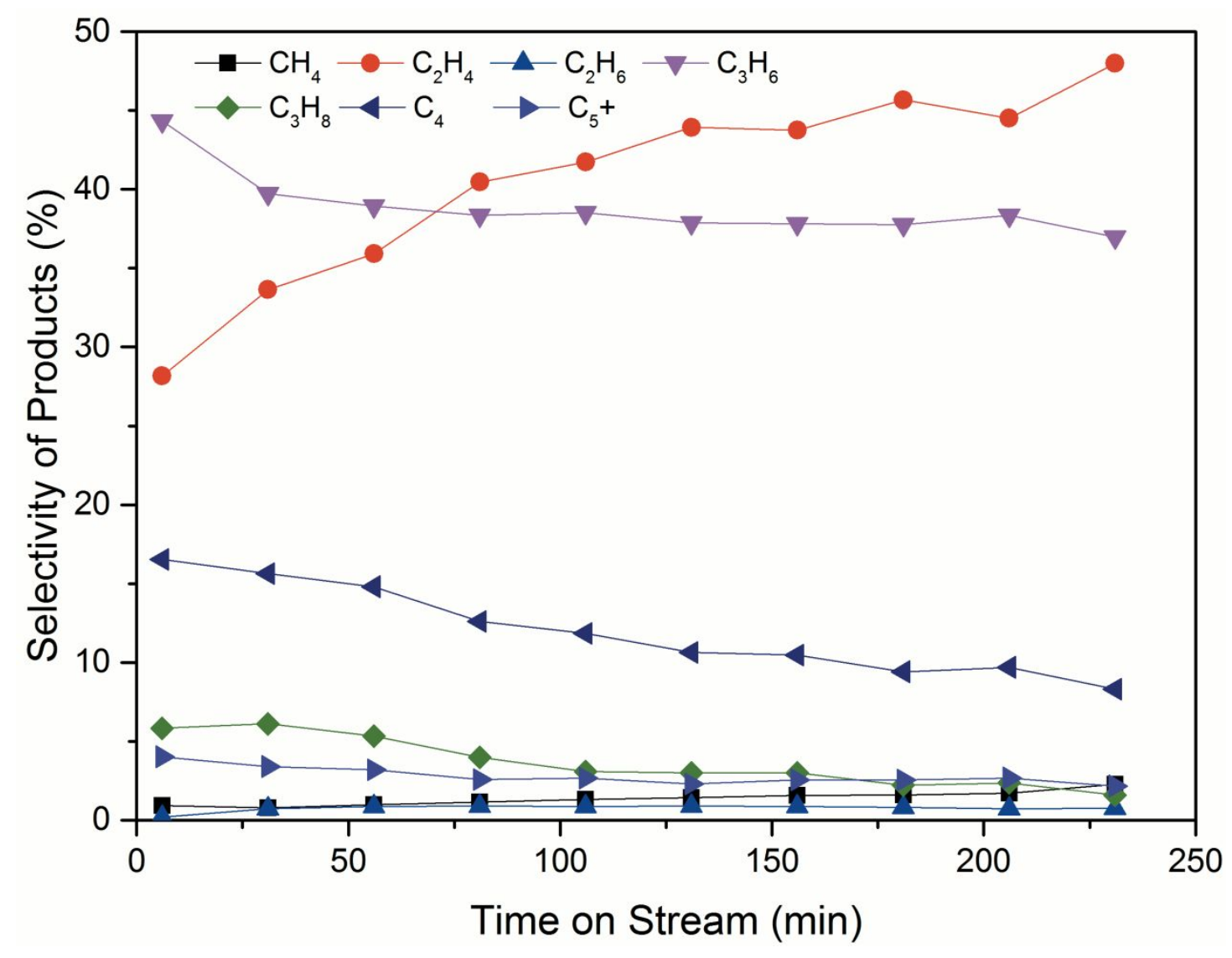

Fig. S5. Selectivity of hydrocarbons over S34-0.08 sample. Experimental conditions: $450{ }^{\circ} \mathrm{C}, \mathrm{WHSV}=2 \mathrm{~h}^{-1}, 50 \%$ methanol solution. 


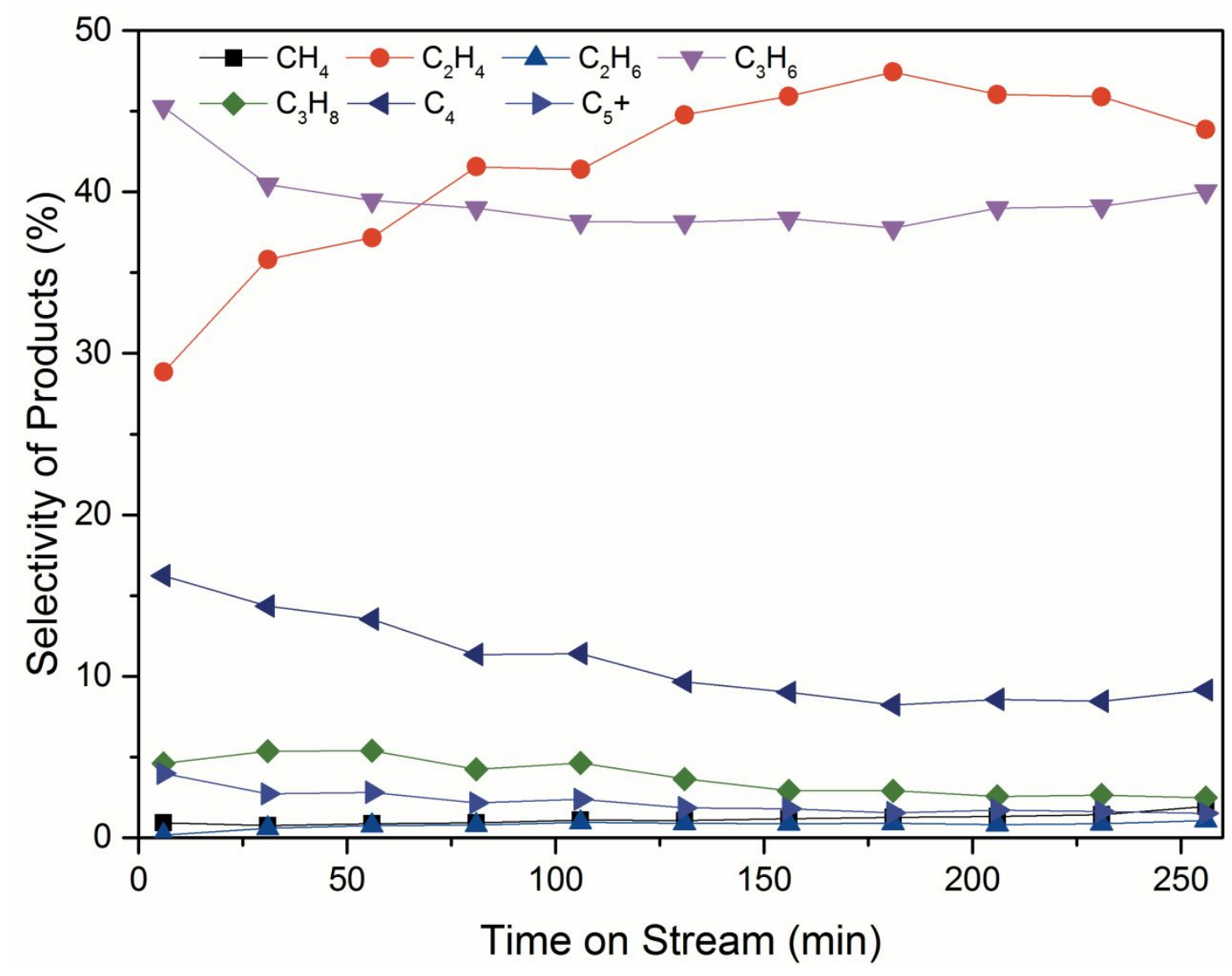

Fig. S6. Selectivity of hydrocarbons over S34-0.12L sample. Experimental conditions: $450{ }^{\circ} \mathrm{C}$, WHSV $=2 \mathrm{~h}^{-1}, 50 \%$ methanol solution. 
Table S1. Selectivity of hydrocarbons over all samples.

\begin{tabular}{|c|c|c|c|c|c|c|c|c|c|}
\hline \multirow{2}{*}{ Samples } & \multirow{2}{*}{$\begin{array}{l}\text { TOS* } \\
(\min )\end{array}$} & \multicolumn{8}{|c|}{ Selectivity (\%) } \\
\hline & & $\mathrm{CH}_{4}$ & $\mathrm{C}_{2} \mathrm{H}_{4}$ & $\mathrm{C}_{2} \mathrm{H}_{6}$ & $\mathrm{C}_{3} \mathrm{H}_{6}$ & $\mathrm{C}_{3} \mathrm{H}_{8}$ & $\mathrm{C}_{4}$ & $\mathrm{C}_{5}^{+}$ & $\mathrm{C}_{2}=+\mathrm{C}_{3}=$ \\
\hline S34-0 & 156 & 1.68 & 44.02 & 0.72 & 32.72 & 1.85 & 10.31 & 2.70 & 82.74 \\
\hline S34-0.04 & 231 & 1.23 & 43.01 & 0.68 & 39.04 & 2.60 & 10.55 & 2.85 & 82.10 \\
\hline S34-0.08 & 181 & 1.55 & 43.75 & 0.86 & 37.81 & 3.00 & 10.47 & 2.56 & 81.56 \\
\hline S34-0.12L & 206 & 1.25 & 47.43 & 0.88 & 37.77 & 2.91 & 8.23 & 1.54 & 85.20 \\
\hline
\end{tabular}

Experimental condition: $50 \mathrm{wt} \%$ methanol, WHSV $=2 \mathrm{~h}^{-1}, \mathrm{~T}=450^{\circ} \mathrm{C}$, catalyst weight $=0.5 \mathrm{~g} ;{ }^{*}$ Lifetime: the reaction duration with the conversion of the methanol $\geqq 98 \%$. 